\title{
Contribution to the wastewater by use of a numerical study of a secondary clarifier comsol multiphysics under
}

\begin{abstract}
The environmental assessment of industrial effluents (containing organic pollutants and minerals without proper treatment), generated by the mineral fertilizer factory and emptied into the seacoast, revealed the concentrations intense of suspended solids (SS).The wastewater treatment is a multi-step process to remove contaminants. At first, large solid particles were removed by sedimentation, flotation and filtration. In a second step, the biological treatment is aggregated by forming the smaller particles, which is called flocs. These flocs can be easily removed by sedimentation. The present investigation studying the separation of water flocs in a circular secondary clarifier. A mixture model is used to model the multiphase turbulent flow in the tank
\end{abstract}

Keywords: comsol, simulation, pollution, clarifier
Volume 4 Issue 2 - 2019

\section{Fares Redouane,' Aissa Abdelrahmane, Bouadi Abed, ${ }^{3}$ Lounis Mourad' \\ 'Laar, University of Science and Technology, Algeria \\ ${ }^{2}$ (LPQ3M), University Mustapha Stambouli Mascara, Algeria \\ ${ }^{3}$ Universitére Ahmed Zabana RELIZANE Center,Algeria}

Correspondence: Dr. Fares Redouane,Ahmed ZABANA University Center of Relizane, Laar, University of Science and Technology, Mohamed Boudiaf, BP I505, Oran, Algeria, Tel 002/377|728504, Email fares-redouane@hotmail.com

Received: December 10,2018 | Published: March 13,2019

\section{Introduction}

Our modern life imposes increasingly important requirements on what to eat, dress and how to manage time and space by finding daily means and methods that are increasingly sophisticated to meet the maximum comfort. ${ }^{1,2}$ Any economic activity caused by the human needs in the entire planet, generates more technological development and human living conditions, pollution problems accompany most of the industrial and agricultural processes including environmental and ecological consequences are very serious and can in some situations simply turn against the objectives initially set by these companies. ${ }^{3,4}$ The degradation of the Earth's environment caused by the intense industrial activities, especially in some developed countries thus enter into the concerns of the nations. Algeria is also affected by pollution in general, and its coast is relatively polluted as it was reported in several research ${ }^{5,6}$ than untreated industrial effluents discharged directly into the Mediterranean sea are the primary causes. ${ }^{7}$ They also showed a growing pollution in the entire Algerian coast, especially in the coastal areas of large cities. Primary causes. ${ }^{7,8}$ They also showed that a growing pollution in the entire Algerian coast, especially in the coastal areas of large cities.

We have tried our best to provide solutions and respond to pollution issues raised during the experimental measurements of physico-chemical parameters features exploiting the numerical simulation tool. ${ }^{5}$ These answers are powerful and efficient, and enable the Arzew Fertial-run business in clean environmental standards. To contributed to reducing pollution after Fertial-Arzew complex, proposed a mathematical model of turbulence ke. To follow the mixing speed, pressure and the phase volume fractions, we propose the application of the mixture model. The latter is a multi phasic flow pattern, particularly suitable for suspensions or mixtures of solid or liquid particles.

\section{Model definition}

By analyzing all released liquids of Fertial-Arzew company directly at its channels evacuations, ${ }^{9}$ it appears to be the the overruns legislative standards. For reasons of efficiency, it is attempted to provide a solution for the parameter measuring solids MY suspension, whose values measured at the three liquid effluent discharge channels exceed a very exaggerated manner standards.

The values from the MES parameter of treated wastewater were significantly lower than those discharged directly into the sea. The wastewater treatment is a process that involves several steps to remove contaminants. The most important of these steps is one in which large solid particles are removed by sedimentation, flotation and filtration obtaining flocs or sludge on the one hand and lightly loaded waste water solids suspension on the other hand. The present investigation studying the separation of water in a circular flocs settling tank used in sewage treatment systems. Figure 1 shows the representative diagram in longitudinal section of the structure of a circular decanter.

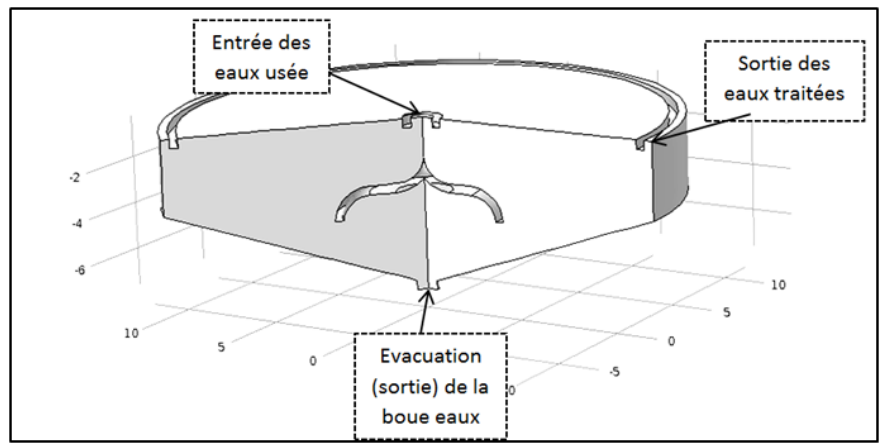

Figure I Longitudinal section diagram showing $2 / 3$ a circular clarifier.

Liquid wastes from Fertial-Arzew company arrive via a channel flowing at the entrance of the clarifier at its center. The treated water leaves at its ends leaving the clarifier decant the bottom solids suspensions. In recent exit through the bottom of clarifier under the effect of gravity to evacuate to dedicated sites or also for a suitable adjustment. To accentuate the wall settling phenomenon («S») is 
provided in the center of the clarifier to prevent the waste water jet arriving through inlet re-mix sludge already settled to the bottom of clarifier.

The clarifier in question has an axial symmetry which allows us to

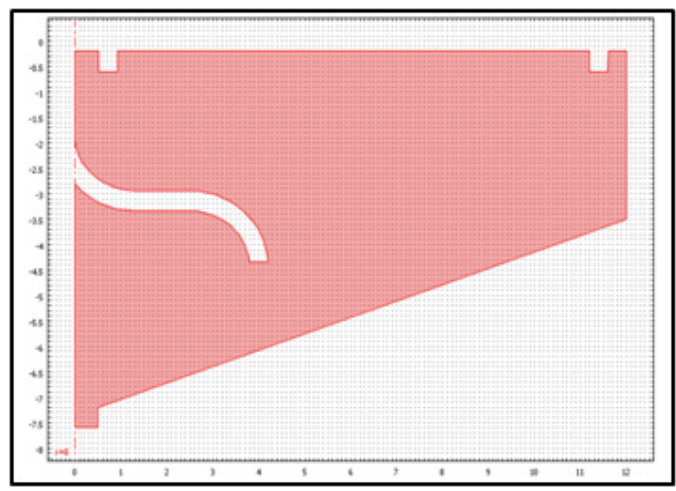

reduce the number calculations operations by merely half the system in 2D, as shown in Figure 2. The first figure shows the axisymmetric diagram of half of the clarifier 2D and the second mesh in its triangular cells.

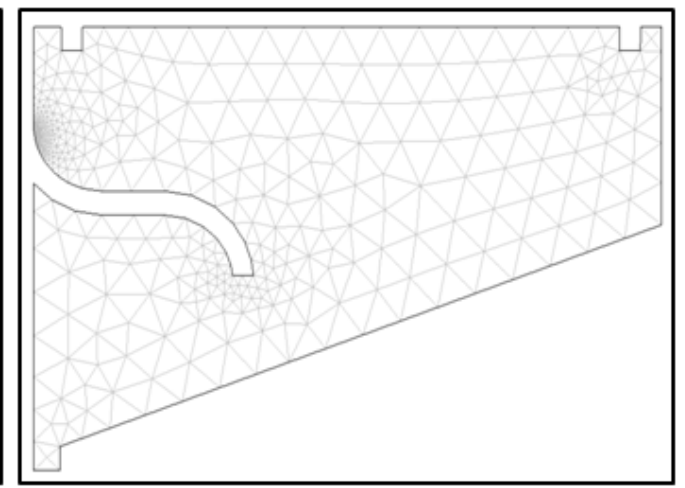

Figure $\mathbf{2}$ Representation of the diagram of axisymmetric geometry and its triangular mesh.

Our model is simulated on a circular secondary clarifier, ${ }^{10}$ the flocs are removed from water by sedimentation and this through gravity. The purpose of this study is to investigate the complex turbulent poly phasic flow in a circular secondary clarifier.

\section{FIG Imontre geometry of the clarifier}

The sludge used, consisting of a mixture of solid flakes and water enters through the inlet of the reservoir. The fuel tank has two outputs. One is located in the center of the tank bottom. The purpose of the outlet port is to remove the flocs sedimented from the reservoir. There is also a peripheral outlet purified water as shown in Figure 2, which also shows the corresponding model in $2 \mathrm{D}$ axisymmetric. We modeled the floc by solid circular of equal size. The model consists of a set of equations, boundary conditions and initial conditions. The set is defined on a geometry decomposed into sub-areas and limited by borders. This model is simulated with COMSOL Multiphysics, or we first made the physical description of the problems (variables, equations, initial limits and conditions), followed by the implementation of our model, and finally made discussions on the results.

\section{Mathematical model}

Based on the conservation of mass and momentum of each phase, the mixture model uses the following equations:

$$
\begin{aligned}
& \rho u_{t}+\rho(u . \nabla) u=-\nabla p-\nabla \cdot\left(\rho c_{d}\left(1-c_{d}\right) u_{s l i p}^{2}\right)+\nabla \cdot \tau_{G m}+\rho g \\
& \left(\rho_{c}-\rho_{d}\right)\left[\nabla \cdot\left(\phi_{d}\left(1-c_{d}\right) u_{s l i p}-D_{m d} \nabla \phi_{d}\right)\right]+\rho_{c}(\nabla \cdot u)=0 \\
& \frac{\partial}{\partial t}\left(\phi_{d} \rho_{d}\right)+\nabla \cdot \phi_{d} \rho_{d} u_{d}=0
\end{aligned}
$$

Where, $u$ represents the velocity of the mixture $(\mathrm{m} / \mathrm{s}), \rho$ is the mixture density $\left(\mathrm{kg} / \mathrm{m}^{3}\right), \mathrm{p}$ is the pressure $(\mathrm{Pa})$, and $\mathrm{Cd}$ is the mass fraction of the solid phase $(\mathrm{kg} / \mathrm{kg})$. In addition, uslip is the relative velocity between the two phases $(\mathrm{m} / \mathrm{s})$, is the sum of the viscous and turbulent stress $\left(\mathrm{kg} /\left(\mathrm{m} \cdot \mathrm{s}^{2}\right)\right)$, and $\mathrm{g}$ is the gravity $\left(\mathrm{m} / \mathrm{s}^{2}\right)$. The mixing speed $(\mathrm{m} / \mathrm{s})$ is defined as $\tau_{G m}$

$$
u=\frac{\phi_{c} \rho_{c} u_{c}+\phi_{d} \rho_{d} u_{d}}{\rho}
$$

Ouet represent respectively the volume fractions of the liquid phase (continuous phase) and the solid phase (discontinuous phase) $\left(\mathrm{m}^{3} / \mathrm{m}^{3}\right)$, the velocity of the liquid phase $(\mathrm{m} / \mathrm{s})$ the speed of the solid phase $(\mathrm{m} / \mathrm{s})$, the density $\rho$ c liquid phase $\left(\mathrm{kg} / \mathrm{m}^{3}\right)$, the density $\rho d$ solid phase $\left(\mathrm{kg} / \mathrm{m}^{3}\right), \rho$ is the density and mixture of $\left(\mathrm{k}_{r} / \mathrm{m}^{3}\right)$. The relationship between the two phases of speeds is defined by $\phi_{c} \phi_{d} u_{c} u_{d}$

$$
u_{c d}=u_{d}-u_{c}=u_{s l i p}-\frac{D_{m d}}{\left(1-c_{d}\right) \phi_{d}} \nabla \phi_{d}
$$

For the solid particles sliding speed, we can use the drag act Hadamard-Rybczynski. In the mode of application of the mixture model of the Hadamard-Rybczynski slip speed is provided as a predefined template. The mode of application then calculates the slip speed as a result:

$$
u_{\text {slip }}=-\frac{\left(\rho-\rho_{d}\right) d_{d}^{2}}{18 \rho \eta} \nabla p
$$

Where denotes the diameter of the solid particles $(\mathrm{m})$. To the mixture density and viscosity, we offer the following expressions $d_{d}$

$$
\eta=\eta_{c}\left(1-\frac{\phi_{d}}{\phi_{\max }}\right)^{-2.5 \phi_{\max }}
$$$$
\rho=\phi_{c} \rho_{c}+\phi_{d} \rho_{d}
$$

The turbulence model k- $\varepsilon$ is used to determine the eddy viscosity. The mode of application calculates the dispersion of particles of Dmd coefficient $\left(\mathrm{m}^{2} / \mathrm{s}\right)$ from

$$
D_{m d}=\frac{\eta_{T}}{\rho \sigma_{T}}
$$

Where $\eta \mathrm{T}$ is the eddy viscosity (Pa.s) and $\sigma \mathrm{T}$ is the (dimensionless) turbulent Schmidt number,

The mass flow of the solid phase through the inlet is given by

$$
M_{\text {in }}=\int_{\text {inlet }}\left|v_{\text {in }}\right| \phi_{\text {din }} \rho_{d} d S
$$

Correspondingly to the flow through the peripheral outlet

$$
M_{\text {out }}=\int_{\text {outlet }}\left|v_{\text {out }}\right| \phi_{\text {dout }} \rho_{d} d S
$$


The difference between the latter two entities provides the remaining mass at the bottom clarifier. Note that the mode of application of the model mix automatically configures all the modeling equations.

\section{Convergence of the solution}

The numerical method is to interpolate the solution on a discretization of the geometry (mesh). A digital solution is acceptable when the convergence criterion is established, that is to say when the numerical result is very close to the exact solution. This is precisely known, it is only available in some cases for simple geometries. Therefore an error estimate is constructed from a Taylor expansion of the second order differential of the equation system operator. Convergence is reached when the estimated error value is less than a threshold value.

The error for the non-linear case is where $\mathrm{N}$ is the number of nodes, $\mathrm{E}$ is the estimation error, and $\mathrm{W}$ is the weight of each node (equal to 1 by default). This value must be less than a factor defined by the user (the default value is 10-6).

$$
e r r=\sqrt{\left(\frac{1}{N} \sum_{i}\left(\frac{\left|E_{i}\right|}{W_{i}}\right)^{2}\right)}
$$

For a time calculation, it is necessary that the solution satisfies the criterion $\mathrm{E}$ at each time step, where $\mathrm{Ai}$ is the absolute tolerance (default 10-3), and Ui is the relative tolerance (default 10-2).

$$
r r=\sqrt{\left(\frac{1}{N} \sum_{i}\left(\frac{\left|E_{i}\right|}{A_{i}+R\left|U_{i}\right|}\right)^{2}\right)}<1
$$

Convergence reflects the adequacy of the numerical solution with the approximate solution of the model. The quality (digital) of the obtained solution and its stability depend on the mesh refinement and time step to satisfy the CFL condition (Courant - Friedrichs -Lewy) presented in the following equation:

$$
C=\frac{\Delta t}{\Delta L} \max \left(\left|\mathrm{v}_{m}\right|\right) \leq 1
$$

Where, $\Delta t$ is the time interval $\Delta L$ is the size range, and vm is the slow component of the velocity.

\section{Conditions to the limits}

Once the geometry of our clarifier system is defined and meshed, it fixed the boundary conditions of the system. After setting the initial mass concentration of the sewage sludge to $0003 \mathrm{Kg} / \mathrm{m}^{3}$, is fed the input speed of the waste water to $1.25 \mathrm{~m} / \mathrm{s}$ and pressure is fixed at the outlet of treated water at atmospheric pressure. Then, we choose the most representative type of flow of what is happening inside the circular clarifier and was chosen in this case to turbulent flow k type $\varepsilon$ multiphasic. Comsol Multiphysics after execution of the computer code on our system that consists of solving the set of equations governing the digital decanter and after verification of convergence curves that provide information on the reliability of the results obtained solutions were recovered in different forms. To demonstrate the appearance phase separation in the decanter under the effect of gravity, the mass concentrations showing the waste water in $\mathrm{Kg} / \mathrm{m}^{3}$ dans the assumed two-phase basin as shown in Figure 3 during the settling period, which lasts for about 24 hours. Can be obtained from solutions of other information on other physical parameters such as speed lines, the current flow lines.

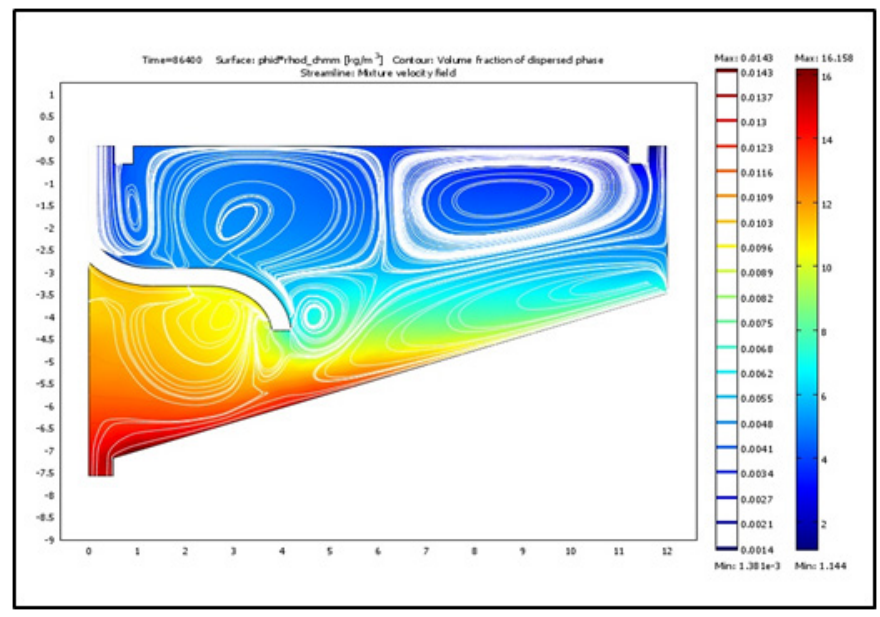

Figure 3 streamlines and concentration of the solid phase during the settling.

\section{Results and discussion}

Figure 3 shows stream lines of the mixing rate and the solid phase concentration, measured in $\mathrm{kg} / \mathrm{m}^{3}$, after 24 hours. At this point, the flow has reached a stable state. We can interpret the speed of the average mixing time. As expected, the solid particles fall down. However, the turbulent fluctuations results in the diffusion of the solid phase have a negative effect on the separation (Figure 4).

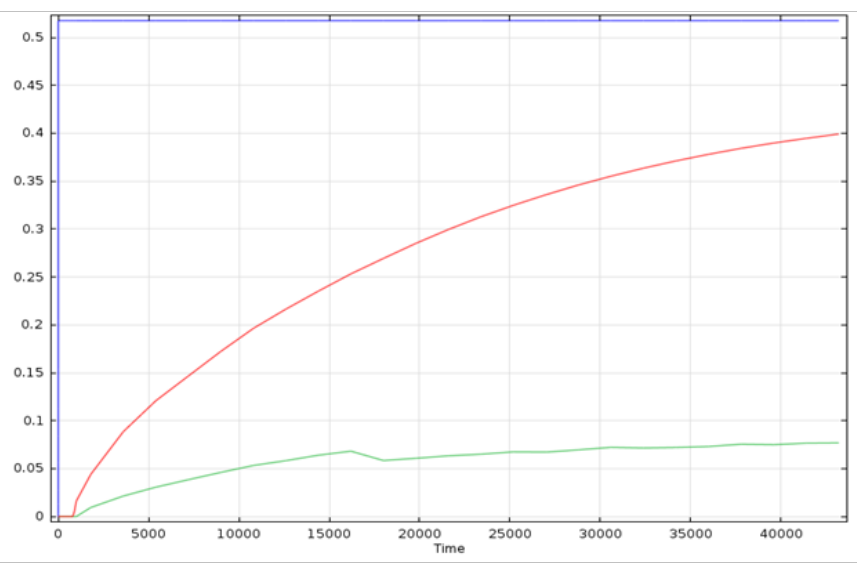

Figure 4 Mass flow of the dispersed phase at the inlet (blue), output device (green) and central outlet (red).

The clarifier and removes solid particles Min rates-Mut. Calculation of the rate of withdrawal from the results shows that the clarifier removes $0518-.151=.367 \mathrm{~kg}$ of solid particles per second.

The results of numerical simulations clearly show the effectiveness of the clarifier, which forces the driven solid particles of gravity to get to the pond bottom, leaving the surface relatively light-weight solid effluent water. Also, it confirms what was reported previously on the importance of the wall introduced into the clarifier, where the impact is observed on device performance by preventing the clearest water from coming into contact with the sludge piled deep. Depending on requirements, we can get more details on a particular region or regions of the decanter to improve and optimize there after the digital system. 


\section{Conclusion}

Marine pollution by wastewater is a serious problem in the Mediterranean Sea. The country's defining reject most of their liquid effluents that this is from the sanitation water towns or discharges from industry without any treatment and Algeria is one. Failure prediction and lack of stations and sewage systems waste water just before the discharge of liquid waste into the sea, causing the deterioration of the overall health and air quality of coastal waters. This causes significant disruption of ecosystems.

Clearances of Fertial-Arzew society as industrial waste water discharged directly into the sea. Although it has the ISO 14001 certificate, the release of this company pollution results of measurements of parameters indicative reveal that study sites are more or less polluted by wastewater discharges. However, prolonged contamination of sea water with these high values of MES up to $260 \mathrm{mg} / \mathrm{L}$ degrade the marine ecosystem and harm wildlife and existing marine flora.

Measurements of MY show the need for pretreatment of raw wastewater before sending them into the sea and improve their quality according to the required standards as well as to protect the environment and human health. To remedy this, we proposed a wastewater treatment system called «clarifier» or «settler», which allows a considerable reduction in the values of MES. The values found by numerical simulation under the calculation code Comsol Multiphysics, clearly showed the improvement of the quality of treated water from those who were not treated at all. The clarifier separates the suspended impurities of clean water. The latter is sent to the sea via the output device and recovered at the bottom outlet of the purification system sediments as sludge. Thus, the sludges are prevented from going contaminate the sea water and can be upgraded later. Our well designed system reduced the amount of suspended solids up to $60 \%$. The fact that contaminated water were retained in the clarifier tank for 24 hours, before sending them into the sea for settling.

\section{Acknowledgments}

None.

\section{Conflicts of interest}

Authors declare that there is no conflict of interest.

\section{References}

1. Teh CY, Wu TY, Juan JC. Potential use of rice starch in coagulationflocculation process of agro-industrial wastewater: Treatment performance and floc characterization. Ecological Engineering. $2014 ; 71: 509-519$

2. Mompoint M. Evaluation of environmental hazards generated by urban liquid effluents on the ecosystem of the bay of Port-au-Prince: First methodological approach. Quisqueya University-Civil Engineer ; 2004.

3. Renuka N, Sood A, Prasanna R, et al. Phycoremediation of wastewaters: a synergistic approach using microalgae for bioremediation and biomass generation. International Journal of Environmental Science and Technology. 2015;12(4):1443-1460.

4. Espinosa F, Garcia-Garcia JM, García-Gómez JC. Sewage pollution and exinction risk: an endangered limpet as bioindicator? Biodiversity and Conservation. 2007;16(2):377-397.

5. Fares R, Lounis M. Determination of the quality of sea waters Arzew, Algeria Gulf. Phytochem \& BioSub Journal. 2017;11(2):110-117.

6. Remili Kerfouf SA. Environmental monitoring and coastal sustainable development (case of Oran coast). Proceedings of the 5th International Symposium on the theme «Energy, Climate Change and Sustainable Development», Tunisia : Hammamet ; 2009.

7. Taghezout Fatima. Environmental impact on the water releases the long littoral Western Algerian, Magister in: environmental sciences, faculty of natural sciences and life biology department laboratory networking environmental monitoring (ERA will) USTO-MB. 2015.

8. Ridha Hachicha. Determination of priority measures for pollution of the coastal area south of Grand Sfax except SIAPE, Scenarios \& remediation Environmental Regulations. Tunisia SMAP III project (2006-2008), Strategy Management Integrated Coastal Zone South Grand Sfax; 2008.

9. Fares R, Louns M. Pollution characterization of liquid wastes of the factory complex Fertial (Arzew, Algeria). J Air Wast Manage Assoc. 2016;66(3):260-266.

10. Comsol Multiphysics User's Guide (c) Copyright 1994-2008. Comsol $\mathrm{AB}$. 